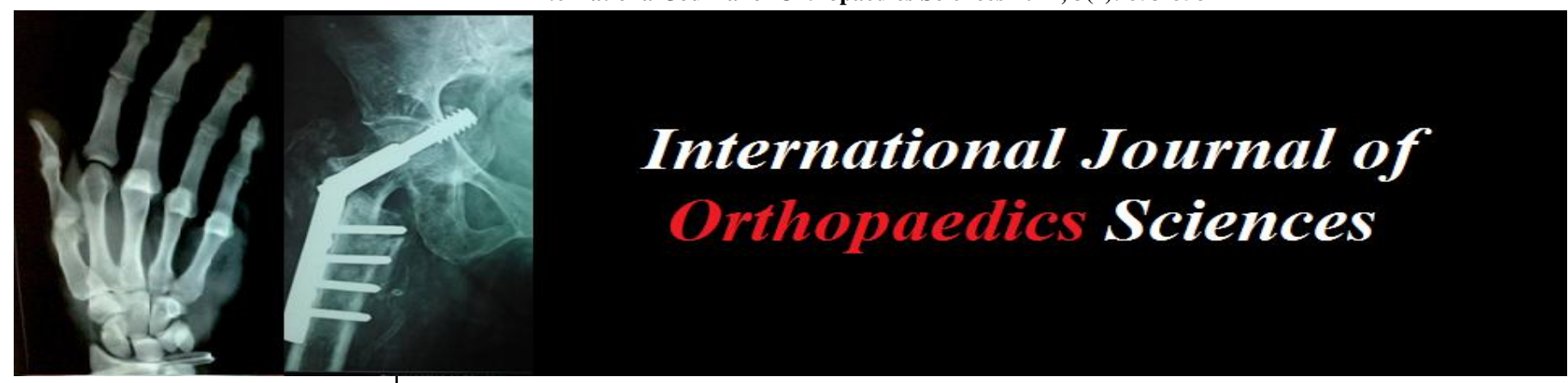

ISSN: $2395-1958$

IJOS 2017; 3(4): 853-858

(C) 2017 IJOS

www.orthopaper.com

Received: 27-08-2017

Accepted: 28-09-2017

Dr. Sandeep Deore

Dr. D. Y. Patil Medical College and Hospital, Nerul, Navi

Mumbai, Maharashtra, India

Dr. Bhushan Patil

Dr. D. Y. Patil Medical College

and Hospital, Nerul, Navi

Mumbai, Maharashtra, India

Dr. Sachin Kale

Dr. D. Y. Patil Medical College

and Hospital, Nerul, Navi

Mumbai, Maharashtra, India

Dr. Prakash Samant

Dr. D. Y. Patil Medical College and Hospital, Nerul, Navi

Mumbai, Maharashtra, India

Dr. Sheran Ali

Dr. D. Y. Patil Medical College and Hospital, Nerul, Navi

Mumbai, Maharashtra, India

Correspondence

Dr. Sandeep Deore

Dr. D. Y. Patil Medical College

and Hospital, Nerul, Navi

Mumbai, Maharashtra, India

\section{Influence of meniscal repair versus meniscectomy on $A C L$ reconstruction in terms of knee stability and radiological imaging}

\author{
Dr. Sandeep Deore, Dr. Bhushan Patil, Dr. Sachin Kale, Dr. Prakash \\ Samant and Dr. Sheran Ali
}

DOI: https://doi.org/10.22271/ortho.2017.v3.i41.117

\section{Abstract}

Objective: To compare effect of meniscus repair and meniscectomy in patients undergoing ACL reconstruction in terms of clinical instability and radiological tunnel widening 1 year after surgery.

Introduction: Meniscectomy has been shown to have detrimental effects on knee in terms of increased contact pressure in femoral and tibial condyles, rotary instability and early changes of osteoarthritis. These changes are exaggerated in ACL deficient knees. Patients present to the surgeon at different times after injury ranging from 3 weeks to one year. Depending upon status of meniscal tears and its reparability at the time of surgery, surgeon has to choose between meniscectomy and meniscus repair during ACL reconstruction. We tried to compare meniscus repair and meniscectomy in terms of knee stability and radiological tunnel widening after ACL reconstruction in short term follow up.

Methodology: 30 patients who underwent ACL reconstruction were selected after going through selection criteria, were divided between meniscus repair group and meniscus excision group. Meniscus tears, which were in the red red zone were repaired with inside out, all inside and outside in methods, depending upon their location from anterior to posterior zones. Those tears which presented late were irreducible, avascular and became loose body scuffing articular cartilage, were excised with shaver. All patients underwent standard ACL reconstruction with hamstring graft. Patients were evaluated clinically and radiologically at 3 months 6 months and 12 months.

Results: Group 1 had 14 patients who underwent meniscus repair and group 2 had 16 patients who underwent meniscectomy during ACL reconstruction. Evaluation with Lachman's test showed that, 14\% patients (2 out of 14) from Group 1 had grade 2 Lachman test. Thirty seven \% of group 2 had grade 2 Lachman test though the difference was not significant $(\mathrm{p}=0.151)$. Tibial tunnel diameter in meniscectomy group increased from $8 \mathrm{~mm}$ to $11.86 \mathrm{~mm}(\mathrm{~S} . \mathrm{D}=1.11$.) at 12 months. Tibial diameter in group 2 increased from $8 \mathrm{~mm}$ to $12.28 \mathrm{~mm}$ (S.D. $=1.52),(\mathrm{p}=0.394)$.

Conclusion: Whenever ACL tear is associated with medial or lateral meniscal tears, meniscal repair is the preferred treatment over meniscectomy. The amount of tunnel widening is higher when meniscus is excised as compared to meniscal repair. The anterior tibial translation remains higher when meniscus is excised during ACL reconstruction, though short term functional results are same.

Keywords: Meniscus repair, meniscectomy, ACL reconstruction, Lachman's test, tunnel widening

\section{Introduction}

Medial and lateral meniscus apart from load sharing have important role in knee kinematics along with other structures of the knee. ACL tear is a common injury in athletes as well as recreational players. The combination of traumatic ACL tear along with meniscal tear has been found from 50 to $68 \%$ by Stergios et al. Conservative treatment of ACL tears has been shown to have fair to poor outcome in up to $87 \%$ patients and they worsen in future to osteoarthritis of the knee. Granan et al has shown that the risk of injury to cartilage increases by $1 \%$ every month after ACL injury if untreated ${ }^{[1]}$. ACL reconstruction has stood test of time to regain knee stability with earlier return to sport and reduce time to regain normal activities. Also early ACL reconstruction is has advantages if associated with meniscal tears. It makes knee more stable and prevent progression of small meniscal tears, meniscal repair can be done in the same surgery and presence of fibrin in blood clot after surgery provide better healing environment for a meniscal tears. 
The delayed intervention after 3 months had higher meniscal injuries as high as $70 \%$ and they were less repairable only upto $17 \%$ [2]. Still many patients opt for conservative treatment like bracing, physiotherapy. If they try to regain high performance activities, they have higher risk of retwisting their knees and damaging their meniscus or cartilage. Also when patients presented late for treatment, meniscal tears get deformed or progressed to complete tears and become loose body ending up in meniscectomy. Follow up as long as 40 years after medial meniscectomy has shown reduced femoro tibial angle leading to varus malalignment and lower flexion range of the knee ${ }^{[3]}$.

Lorbach et al has shown that medial meniscectomy increases anterior tibial translation in ACL deficient knees by significant amount by Lorbach et al. [4, 5] Total lateral meniscectomy or its untreated root tear is also associated with increase posterolateral rotary instability postoperatively due to its anatomical proximity to popliteus tendon and posterolateral capsule ${ }^{[6]}$. Meniscus repair has higher healing potential. Second look arthroscopy by Matsushita et al showed complete meniscus tear healing in $56 \%$ patients ${ }^{[7]}$.

After ACL reconstruction surgery bony tunnel enlargement is a known complication and it has been associated with anterior laxity in significant number of patients ${ }^{[8]}$. Has never been discussed. We tried to evaluate association of meniscectomy during ACL reconstruction with knee instability and tunnel widening in our study and compare with those cases where meniscus repair was done along with ACL reconstruction.

\section{Materials and methods}

Our retrospective study included 30 patients who were operated within a period from May 2013 to May 2016 at our institute. Patients presented at different follow up time to us ranging from 3 weeks to 1 year.

Inclusion criteria was: 1) Complete ACL tear with medial or lateral meniscus tear. 2) Young physically active male or female age group 18 to 50 years. 3) Patient willing to give consent for the study. 4) Patient willing to undergo rehabilitation after surgery. Exclusion criteria: 1) Medial compartment osteoarthritis, 2) Associated multiligament injury, 3) Associated chondral injury and 4) Revision ACL reconstruction.

All patients were clinically evaluated by Lachman's test, Drawer test, Pivot shift test and Mc Murray's test. After clinical and radiological confirmation, they underwent arthroscopic surgery by single surgeon at our institute. During arthroscopy, the meniscus tear was evaluated for reparability. Grade 3 tears (Fig 1, Fig 2) which are in a vascular red red or red white zone were included in group 1 and were repaired. All inside, inside out and outside in methods were utilized depending on location of the tear (Fig 3). For inside out repair of medial meniscus, safety incision was taken posteromedially to protect sephanous nerve. During lateral meniscus repair, posterolateral safety incision was used to protect peroneal nerve and popliteal vessels. Irreparable, deformed and irreducible tears in avascular zone were trimmed with shaver to achieve stable meniscus rim. In some cases, notchplasty was done if osteophytes were found in femoral notch (Fig 4). They were included in group 2. Independent of meniscal procedure, all ACL tears were reconstructed with four folds $8 \mathrm{~mm}$ semitendinosus graft. Accessory medial portal technique was utilized for ACL reconstruction. Femoral fixation in all patients was extracortical fixation (endobutton with a fixed polyster loop or adjustable loop). On tibial side fixation was either with a suture disc or an interference screw depending upon the length of the graft. Patient's knee was immobilized with a long leg knee brace.

After surgery, 30 patients were divided into two groups. Group 1 had ACL tear with repairable meniscus tear and group 2 had ACL tear with irreparable meniscus tear.

\section{Postoperative protocol}

Postoperatively, static quadriceps strengthening, ankle toe pumps and hip strengthening exercise were started. After two weeks, gradual knee range of motion was started. Group 1 patients were kept non weight bearing for 3 weeks followed by partial weight bearing. At the end of one month, partial weight bearing was done with walker and knee brace up to four weeks. Physiotherapy protocol was similar for group 2 patients except that partial weight bearing was started after 2 weeks. As patients started gaining good quadriceps strength they were asked to walk full weight bearing with hinged knee brace. Full range of motion was achieved till 6 weeks. Third month after the surgery, patients were asked to resume their daily routine activities like walking, stair climbing and jogging. Patients were asked to resume sports activities six to eight months after surgery.

Functional scoring was done using IKDC and Lysholm scoring systems preoperatively and during follow up. At the end of 6 months, objective assessment of knee stability was with Lachman's Test, Drawer's Test and Pivot Shift test.

\section{Radiographic evaluation}

Radiographic evaluation was done at. Anteroposterior and lateral radiographs of the operated knees were taken at every follow up of three months, six months and one year follow up. CT scan was taken during follow up period to estimate amount of tunnel widening (Fig 6).

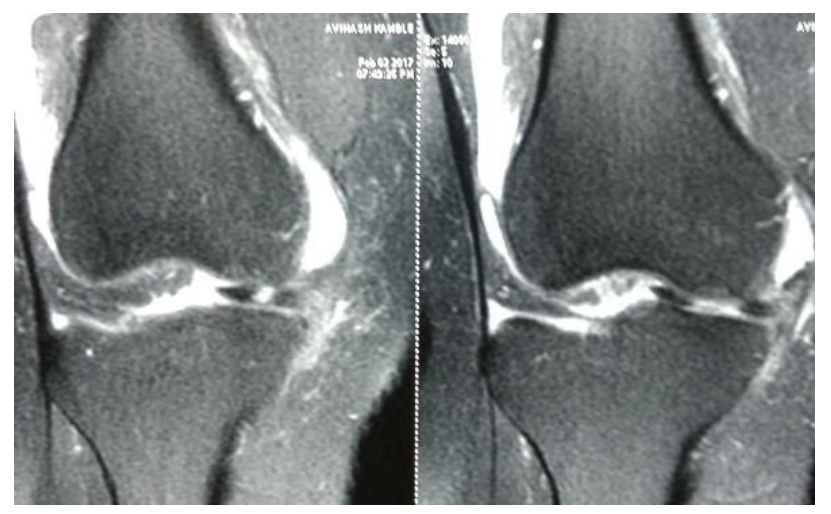

Fig 1: Preop MRI coronal image: displaced medial meniscus tear.

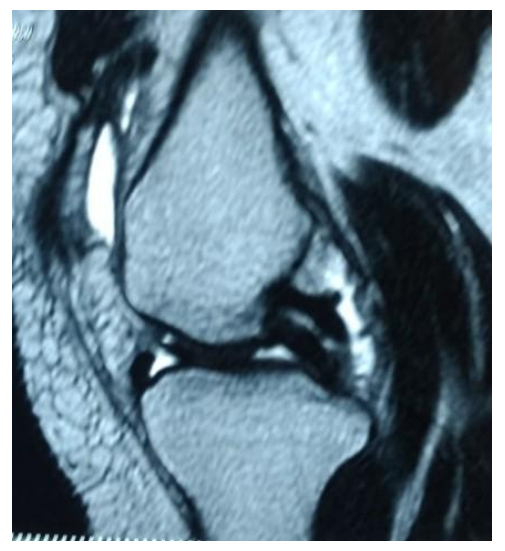

Fig 2: Preop MRI showing double PCL sign in T2 sag indicating bucket handle tear. 


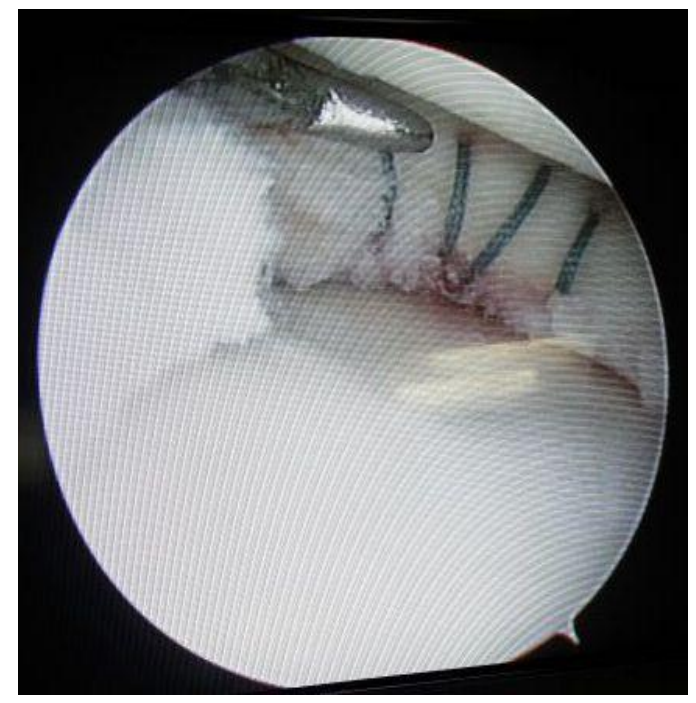

Fig 3: Medial meniscus repair by Inside Out method.
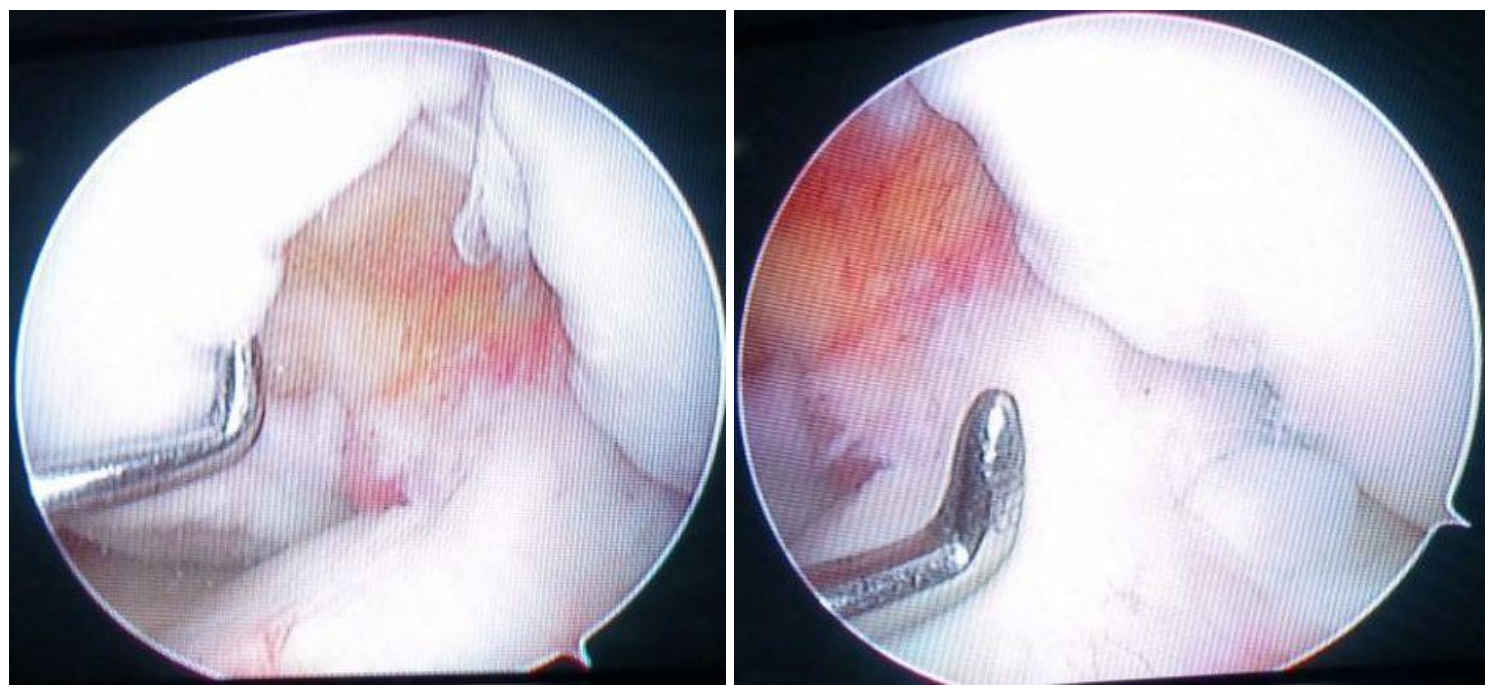

Fig 4: Irreparable chronic medial meniscus tear with early osteophytes in femoral notch inyoung ACL deficient knee.

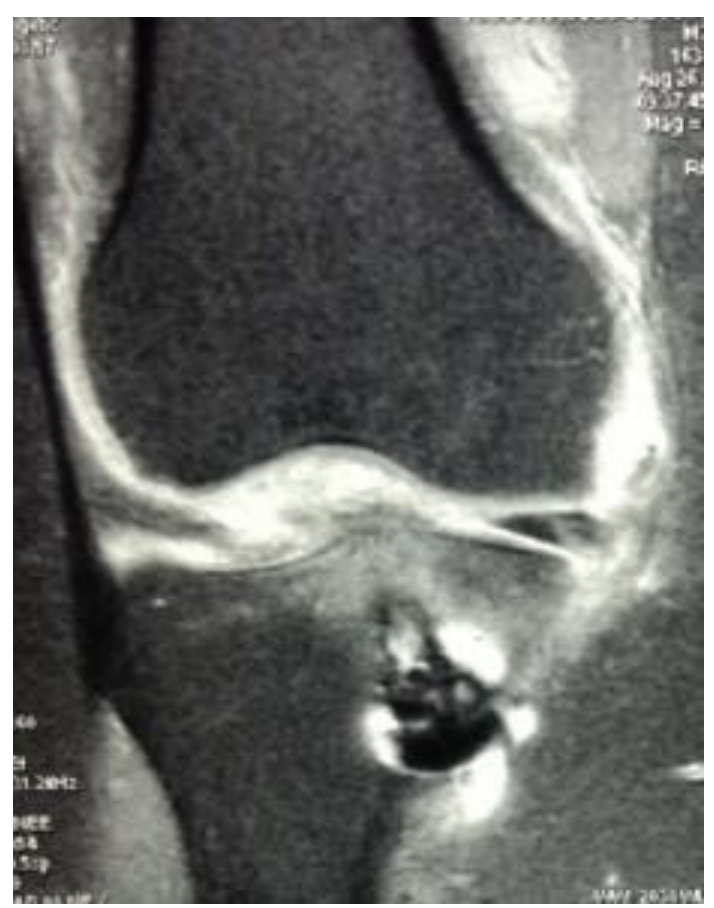

Fig 5: 6 months follow up MRI showing completely healed medial meniscus repair.

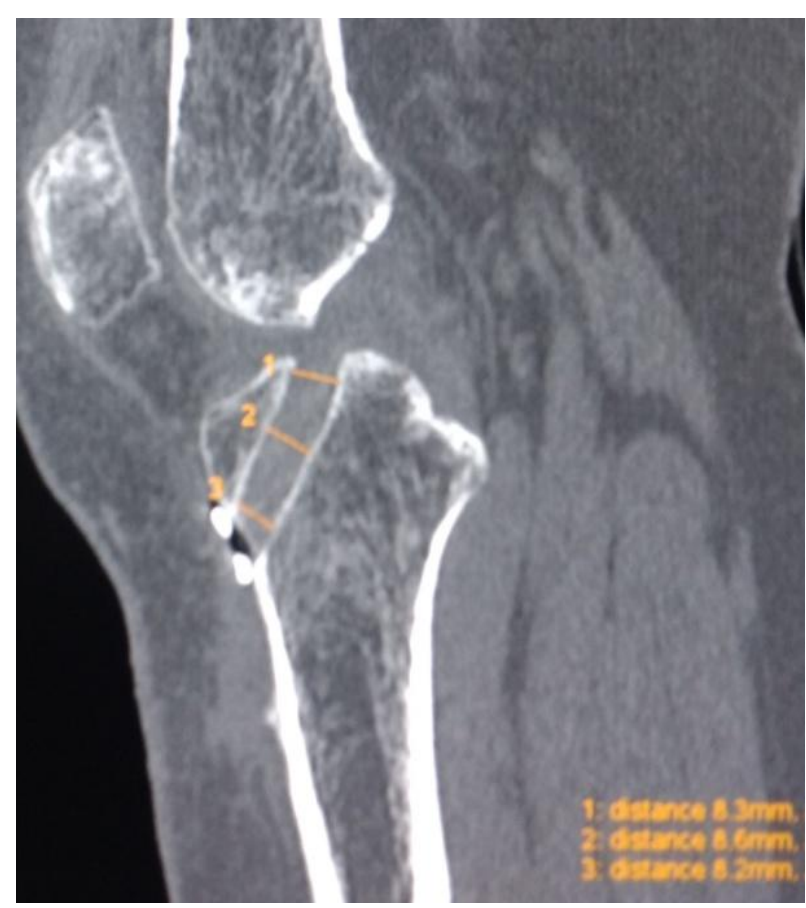

Fig 6: 6 months follows up CT scan sagital section showing tibial tunnel widening. 


\section{Results}

Out of 50 patients, 20 patients had isolated meniscus or isolated ACL tears and hence they had to be excluded from the study. Thus total 30 patients were included in the study. Mean age of the patient was 24.71 years (Range20 to 44 years). There were 28 male and 2 female patients. Out of 30 knees 12(40\%) were right and $18(60 \%)$ were of left side. Seventeen $(56 \%)$ of these patients had sport related injury and $13(44 \%)$ had domestic fall or road accidents. Seven patients had associated lateral meniscus injury and 8 had medial meniscus injury. ACL reconstruction was done with 4 fold hamstring graft with suspensory fixation on femoral side in all cases. On tibial side suture disc was used in 17 patients and interference screw was used in 13 patients.

They were divided into 2 groups. Group 1 had ACL tear with meniscus repair and group 2 had ACL tear with meniscus excision. Group 1 had 14 patients and Group 2 had 16 patients. All patients were called after 3 months, 6 months and 12 months for clinico-radiological evaluation. Tunnel diameters were noted for tibia and femur in coronal, sagital and axial cuts of CT scan. Measurements were taken with digital scales to eliminate magnification bias both on $\mathrm{x}$ rays and CT scans.

Tibia tunnel diameter was calculated at mouth, center and exit of the tunnel on the bone surface both in AP and lateral views. Femoral tunnel diameter was measured at the mouth or intra articular end of the tunnel.

Follow up MRI scan was done after 6 months to assess healing status of the meniscus and ACL graft status (Fig.5). Meniscus was considered to be healed if they were grade 1 or no signal intensity on T2 sections. Meniscus was considered as not healed if there was grade 3 signal intensity.

\section{Tunnel diameter}

Tibial tunnel widening was calculated in both the groups at the end of 12 months. Tibial tunnel diameter in meniscus repair group (Group 1) increased from $8 \mathrm{~mm}$ to $11.86 \mathrm{~mm}$ at 12 months. Tibial diameter in meniscectomy group (Group 2) increased from $8 \mathrm{~mm}$ to $12.28 \mathrm{~mm}$. This was not significant by Student T Test $(p=0.394)$.

Tibia I: Tunnel Diameter in different groups

\begin{tabular}{|c|c|c|c|c|}
\hline Group & No. of patients & Mean & Standard Deviation & P value \\
\hline Group 1 (Meniscus repair) & 14 & 11.86 & 1.11 & 0.394 (NS) \\
\hline Group 2 (Meniscectomy) & 16 & 12.28 & 1.52 & T test \\
\hline
\end{tabular}

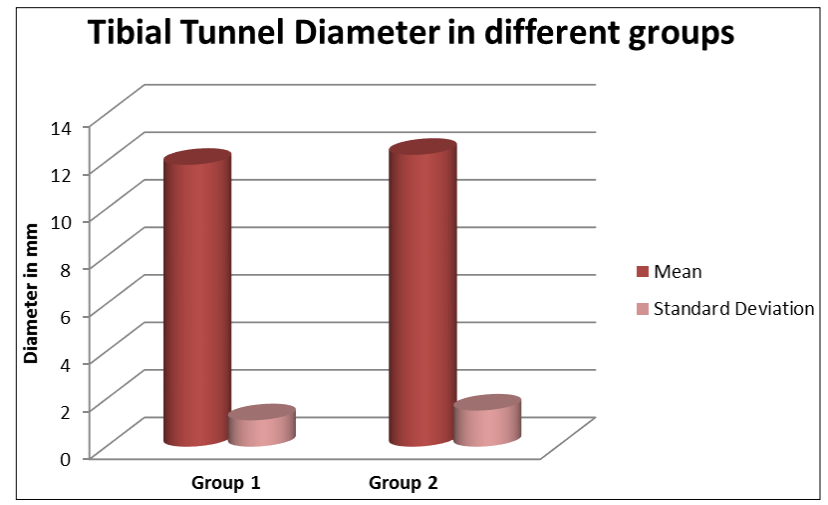

Lachman Test in Different Groups

\begin{tabular}{|c|c|c|c|}
\hline \multirow{2}{*}{ Diagnosis } & \multicolumn{2}{|c|}{ Lachman Test } & \\
\cline { 2 - 3 } & Grade 1 & Grade 2 & P value \\
\hline Group 1 = 14 & $12(85.7 \%)$ & $2(14.2 \%)$ & 0.151 (NS) \\
\hline Group 2 $=16$ & $10(62.5 \%)$ & $6(37.5 \%)$ & Chi Square test \\
\hline
\end{tabular}

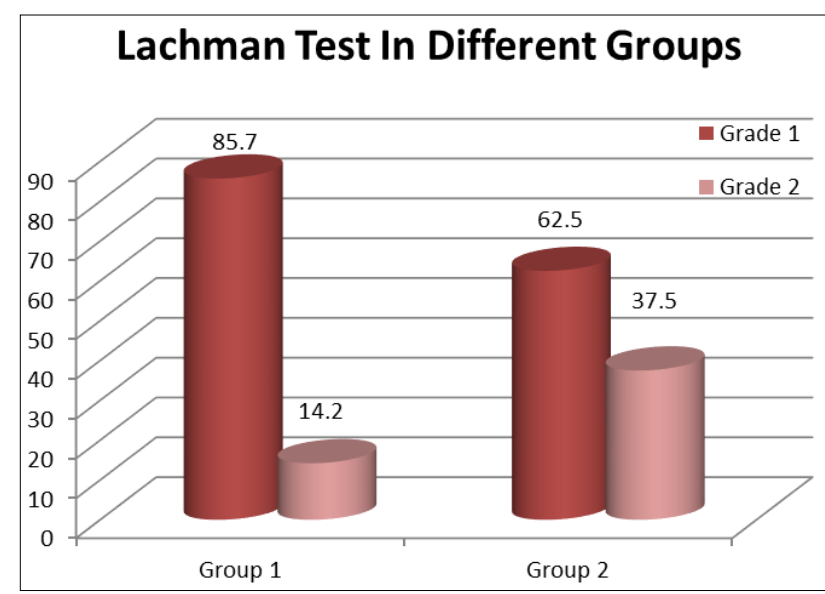

Fourteen percent (2 out of 14) patients from group 1 had grade 2 Lachman's test. Thirty seven \% of group 2 had grade 2 Lachman's test though the difference was not significant ( $p$

\section{$=0.151)$}

Discussion

Importance of intact ACL and meniscus in knee kinematics has been studied in depth in past. ACL gives stability to the knee in coronal plane as well as against the rotational forces. Menisci also have important functions in the knee joint. Apart from basic function like, shock absorption, increasing joint congruity and sharing body weight, menisci also contribute mechanical stability of the knee along with cruciate ligaments especially rotary stability ${ }^{[9]}$.

Conservative treatment of ACL tears has been shown to have fair to poor outcome in upto $87 \%$ patients. Return to work or sports after rehabilitation is as low as $6 \%$. Also $33 \%$ who returned to sport had lower performance level ${ }^{[10]}$.

ACL reconstruction has stood test of time to regain knee stability with earlier return to sport and reduce time to regain normal activities. Still many patients opt for conservative treatment like bracing and physiotherapy. If they try to regain high performance activities, they have higher risk of retwisting their knees and damaging their meniscus or cartilage. The combination of these traumatic ACL tear along with meniscal tear has been found from 50 to $68 \%{ }^{[11]}$. When present together, they can significantly disable both sportsmen as well as nonsportsmen.

Lot of importance has been given to meniscus repair recently 
due to better understanding of anatomy, better fixation methods and refinement in implant technology. Still there are some instances in irreparable tears where meniscus shaving is the treatment of choice. Those menisci which cannot be repaired are treated with excision and stabilization of the rim. Those which are repairable are treated with meniscus repair by different techniques.

Though, untreated meniscal injuries after ACL reconstruction doesn't have poor short term functional scores up to 3 years, the long term prognosis is not good ${ }^{[12]}$. They have higher incidence of osteoarthritis. Lohmander et al in his study in young women soccer players in 12 year follow up has shown that, untreated ACL injuries with clinical laxity have been found to have very high prevalence of pain, functional limitations and radiographic changes of osteoarthritis ${ }^{[13]}$. We had similar findings in our study where a 37 year female with chronic irrepairable meniscus tear had developed osteophytes in the femoral notch (fig 4) where notch plasty was performed after meniscectomy. ACL reconstruction has been shown to reduce the risk of $\mathrm{OA}$ to good extent. In addition, initial injuries associated with ACL tears like meniscal tears, chondral injuries seem to have significant impact on progression to $\mathrm{OA}^{[14]}$. It has been shown that the incidence of meniscal and chondral injuries increases with time after ACL injury. Granan et al has shown that the risk of injury to cartilage increases by $1 \%$ every month after ACL injury ${ }^{[1]}$.

There is a consensus on early reconstruction of ACL. Hur et al shown that early reconstruction of ACL gave higher chances of early meniscal repair with good healing rates than late repairs. The delayed intervention after 3 months had higher meniscal injuries as high as $70 \%$ and they were less repairable only upto $17 \%$. Also when patients presented late for treatment, meniscal tears were deformed or progressed to complete tears and became loose body. Those tears had to be excised. There is also evidence to stress that early ACL reconstruction can regain good stability, knee motion muscle power and postural control ${ }^{[15]}$.

Dilworth et al showed that meniscal tears with ruptured ACL if repaired have been found to have higher healing potential than the isolated meniscal tears ${ }^{[16]}$. The proposed reasons are 1) reconstructed ACL gives better biomechanical stability to the knee avoiding repeat injury to the meniscus, 2) presence of fibrin clot in ACL tears provides better nurturing environment to meniscal repair than the isolated meniscal tears.

The function of the meniscus in the knee joint has been discussed enough in the literature. Menisci act as joint filers and increase the congruity of femoro- tibial surface sharing 40 to $60 \%$ body weight. They have other accessory function like prevention of capsular impingement during knee movement, distribution of synovial fluid to cartilage for nutrition. They also contribute mechanical stability of the knee along with cruciate ligaments specially rotary stability giving additional gliding function to the hinge type of knee joint ${ }^{[9]}$. Meniscectomy has bad influence on joint alignment, load distribution and anterior instablilty ${ }^{[3,4,5]}$.

Medial meniscectomy increases tibio femoral contact stresses by $100 \%$ whereas lateral meniscectomy increases contact stresses by $200 \%$ to $300 \%$. A single radial cut is equivalent to meniscectomy as it cuts off the hoof stresses across the circumferential fibers of the meniscus. Medial meniscectomy has been found to increase anterior tibial translation in ACL deficient knees by Lorbach et al. [4] Total lateral meniscectomy is also associated with increase posterolateral rotary instability postoperatively due to its anatomical proximity to popliteus tendon and postero lateral capsule. Laprade et al has shown the importance of lateral meniscus in ACL reconstructed knees. They found increased anterior translation and rotatory instability of the knees due to posterior lateral meniscal root disruption if they are left untreated after ACL reconstruction ${ }^{[6]}$. This in turn may increase strain on ACL graft. But association of meniscectomy during ACL reconstruction and tunnel widening has never been discussed. We tried to evaluate the same in our study.

Meniscal tears have been classified by Harner et al into three types depending on their location into red zone, red- white and white zone. This classification can be used to predict healing potential of the tear after repair ${ }^{[9]}$. The tears in peripheral vascular zones have higher healing potenetial. Second look arthroscopy by Matsushita et al showed complete meniscus tear healing in $56 \%$ patients ${ }^{[7]}$. Refreshening of RAMP lesion after ACL reconstruction has been described by Yang et al and has shown comparable results with all inside technique. This may be because there are higher healing potential for these peripheral tears as they are in vascular zone. Therefore, meniscus preservation has been given lot of importance in recent times and preferred over meniscectomy procedures.

Still in certain situations when patients present late, these tears are irreparable, deformed and avascular and can damage intact articular cartilage. In these situations, partial or subtotal meniscectomy is a reasonable option for the surgeon. Total meniscectomy exposes vascular peripheral bed for regeneration, while subtotal meniscectomy doesn't regenerate if it's in avascular zone.

Bony tunnel enlargement is a known complication after ACL reconstruction surgery. Webster et al found significant correlation between tibial tunnel enlargement and anterior knee laxity ${ }^{[8]}$. Bone tunnel widening is more associated with many factors like type of graft, graft fixation systems elastic or rigid, single or double bundle fixation, and aggressive rehabilitation ${ }^{[18]}$. The biological theory states that release of $\mathrm{TNF}$, interleukins, and nitric oxide present in the synovial fluid can cause osteolysis. The mechanical reasons for tunnel widening are graft tunnel micro motion in suspensory fixation, stress shielding proximal to the interference screw and nonantomical graft fixation ${ }^{[18,19,20]}$. As menisci along with cruciate ligaments also has important role in biomechanical stability, we have tried to evaluate incidence of tunnel widening and knee instability after ACL reconstruction and compare them in two groups: those with meniscal repair and those with without repair.

In our study, we have found that, the amount of tibial tunnel widening was less i. e. $11.86 \mathrm{~mm}(\mathrm{~S} . \mathrm{D}=1.11$.) in the meniscus repair group than the meniscectomy group 12.28 $\mathrm{mm}$ (S.D. $=1.52$ ), though statistically not significant $(p=0.394)$. The Lachman's Test was grade 2 in $37.5 \%$ of meniscectomy group vs. $12.2 \%$ in meniscus repair group ( $p=$ 0.151 ). Though the functional scores didn't very much, it may be significant on long term follow up.

Drawback of the study was smaller sample size and relatively short follows up period. Also late functional results were better in sportsmen than the normal population as sportsmen were more motivated to regain high demand activities.

\section{Conclusion}

Whenever ACL tear is associated with medial or lateral meniscal tears, meniscal repair should be the first choice. The amount of tibial tunnel widening is higher when meniscus is 
excised as compared to meniscal repair. The anterior tibial translation remains higher when meniscus is excised during ACL reconstruction. Though short term functional results are same in both the groups, randomized controlled trials with larger sample size and longer follow up will have better insight on this subject.

\section{References}

1. Lars-Petter Granan. Timing of Anterior Cruciate Ligament Reconstructive Surgery and Risk of Cartilage Lesions and Meniscal Tears A Cohort Study Based on the Norwegian National Knee Ligament Registry. American journal of Sports Medicine. 2009; 37(5):955-961.

2. Papastergiou SG, Koukoulias NE, Mikalef P. Meniscal tears in the ACL-deficient knee: correlation between meniscal tears and the timing of ACL reconstruction. Knee Surg Sports Traumatol Arthrosc. 2007; 15(12):1438-44.

3. Pengas IP, Nash W, Khan W. Coronal Knee Alignment 40 Years after Total Meniscectomy in Adolescents: A Prospective Cohort Study. Open Orthop J. 2017; 30(11):424-431.

4. Lorbach O, Kieb M, Herbort M. The influence of the medial meniscus in different conditions on anterior tibial translation in the anterior cruciate deficient knee. Int Orthop. 2015; 39(4):681-7.

5. Zaffagnini S, Signorelli C, Bonanzinga. Does meniscus removal affect ACL-deficient knee laxity? An in vivo study. Knee Surg Sports Traumatol Arthrosc. 2016; 24(11):3599-3604.

6. Frank JM, Moatshe G, Brady AW, Dornan GJ, Laprade RF. Lateral Meniscus Posterior Root and Meniscofemoral Ligaments as Stabilizing Structures in the ACL-Deficient Knee: A Biomechanical Study. Orthop J Sports Med. 2017; 15;5(6):2325.

7. Matsushita T, Nagai K, Araki D. Factors associated with the status of meniscal tears following meniscal repair concomitant with anterior cruciate ligament reconstruction. Connect Tissue Res. 2017; 58(3-4):386392.

8. Webster KE, Chiu JJ, Feller JA. Impact of measurement error in the analysis of bone tunnel enlargement after anterior cruciate ligament reconstruction. Am J Sports Med. 2005; 33:1680-1687.

9. Campbell's Operative Orthopedics, Eleventh Edition, part XIII, Chapter 43, Knee Injuries.

10. Robert Barrack 1. The Outcome of Nonoperatively Treated Complete Tears of the Anterior Cruciate Ligament in Active Young Adults, Clinical Orthopedics and Related Research, 1990, 259.

11. Stergios Papastergiou G. Meniscal tears in the ACLdeficient knee: Correlation between meniscal tears and the timing of ACL reconstruction Knee Surg Sports Traumatol Arthrosc. 2007; 15:1438-1444.

12. Singh A, Wei DT, Lin CTP, Liang S, Krishna L. Concomitant meniscal injury in anterior cruciate ligament reconstruction does not lead to poorer shortterm post-operative outcomes. Knee Surg Sports Traumatol Arthrosc. 2017, 15.

13. Lohmander LS. High Prevalence of Knee Osteoarthritis, Pain, and Functional Limitations in Female Soccer Players Twelve Years after Anterior Cruciate Ligament Injury, Arthritis \& Rheumatism. 2004; 50(10):31453152.

14. Nikolaos Paschos K. Anterior cruciate ligament reconstruction and knee osteoarthritis World J Orthop. 2017; 18;8(3):212-217.

15. Hur CI, Song EK, Kim SK, Lee SH, Seon JK. Early anterior cruciate ligament reconstruction can save meniscus without any complications. Indian $\mathbf{J}$ Orthop. 2017; 51(2):168-173.

16. Dilworth W. The incidence of healing in arthroscopic meniscal repairs in anterior cruciate ligamentreconstructed knees versus stable knees. American journal of sports medicine. 1992; 20:2.

17. Yang J, Guan K, Wang JZ. Clinical study on the arthroscopic refreshing treatment of anterior cruciate ligament injury combined with stable medial meniscus ramp injury. J Musculoskeletal Neuronal Interact. 2017; 1;17(2):108-113.

18. Zijl JA, Kleipool AE, Willems WJ. Comparison of tibial tunnel enlargement after anterior cruciate ligament reconstruction using patellar tendon autograft or allograft. Am J Sports Med. 2000; 28(4):547-51.

19. Barber FA, Spruill B, Sheluga M. The effect of outlet fixation on tunnel widening. Arthroscopy. 2003; 19(5):485-92.

20. Zysk SP, Fraunberger P, Veihelmann A, Dörger M, Kalteis $\mathrm{T}$, Maier $\mathrm{M}$ et al. Tunnel enlargement and changes in synovial fluid cytokine profile following anterior cruciate ligament reconstruction with patellar tendon and hamstring tendon autografts. Knee Surg Sports Traumatol Arthrosc. 2004; 12(2):98-103. 